Check for updates

Cite this: RSC Adv., 2019, 9, 13722

\title{
A circular RNA circ_0000115 in response to graphene oxide in nematodes $\uparrow$
}

\author{
Lifang Shi, Xiaohuan Jia, Tiantian Guo, Lu Cheng, Xiaoxiao Han, Qiuli Wu (DD* \\ and Dayong Wang $\mathbb{D}^{*}$
}

Circular RNAs (circRNAs) play important roles in regulating various biological processes; however, their roles in regulating the toxicity of engineered nanomaterials (ENMs) are still unclear. Based on Illumina HiSeq2500 sequencing, we here identified 43 dysregulated circRNAs in graphene oxide (GO) (1 mg L $^{-1}$ ) exposed nematodes. Five of these candidate circRNAs could be further dysregulated by GO exposure in the range of $\mu \mathrm{g} \mathrm{L}^{-1}$. Using the RNA interference (RNAi) technique, we found that the alteration in expressions of circ_0000115, circ_0000247, and circ_0000665 mediated a protective response to GO exposure; however, the alteration in expressions of circ_0000201 and circ_0000308 mediated the toxicity induction of GO. In nematodes, the circ_0000115 acted in certain tissues (intestine and neurons) to regulate GO toxicity. Moreover, an intermediate filament protein IFC-2 required for intestinal development was identified as a target of circ_0000115 in regulating the GO toxicity. In the intestine, intestinal IFC-2 acted further upstream of FOXO transcriptional factor DAF-16 in the insulin signaling pathway to regulate the GO toxicity. Therefore, intestinal circ_0000115 in the signaling cascade of circ_0000115-IFC-2-DAF-16 regulates the GO toxicity by modulating the function of IFC-2.

Received 7th February 2019

Accepted 29th April 2019

DOI: 10.1039/c9ra00997c

rsc.li/rsc-advances elegans. ${ }^{17}$ It has been shown that the circRNAs are involved in the regulation of many biological processes, including stress response and human diseases. ${ }^{18-21}$ However, the potential roles of circRNAs in the regulation of ENMs toxicity are still largely unclear.

Classic model animal C. elegans has been widely used in the field of life sciences. ${ }^{22}$ Meanwhile, due to its sensitivity to environmental toxicants, C. elegans has been employed as an important in vivo assay system for the study of toxicity assessment or toxicological mechanisms of certain environmental toxicants. $^{23-27}$ Recently, it has been shown that C. elegans is useful for nanotoxicological study of different ENMs, including carbon-based ENMs. ${ }^{28-43}$ In nematodes, GO exposure could cause toxicity on the functions of both primary targeted organs, such as intestine, and secondary targeted organs, such as reproductive organs and neurons..$^{30,44-47}$ In the genome of $C$. elegans, at least mitogen-activated protein kinases (MAPKs), insulin, Wnt, cell death and DNA damage signaling pathways have been identified to be required for the control of response of animals to GO exposure. ${ }^{45,48-52}$ The main aim of this study is to further determine the roles and the functions of circRNAs in the regulation of GO toxicity using in vivo assay system of $C$. elegans. We discussed the potential implications of the dysregulation of some circRNAs in the nanosafety assessment in GO exposed nematodes. Moreover, we focused on one of the dysregulated circRNAs, circ_0000115, to examine the underlying molecular mechanism for its role in the regulation the GO toxicity. With the circ_0000115 as an example, we identified the
Key Laboratory of Environmental Medicine Engineering in Ministry of Education, Medical School, Southeast University, Nanjing 210009, China. E-mail: qlwu@seu. edu.cn; dayongw@seu.edu.cn

$\dagger$ Electronic supplementary information (ESI) available. See DOI: 10.1039/c9ra00997c 
mechanism for a circular RNA in response to graphene oxide in organisms.

\section{Materials and methods}

\subsection{Preparation and characterization of GO}

GO was prepared from natural graphite powder according to a modified Hummer's method. GO was finally obtained by ultrasonication of as-made graphite oxide in water. Stock solution of GO $\left(1 \mathrm{mg} \mathrm{mL}^{-1}\right)$ was prepared in $\mathrm{K}$ medium by sonication for $30 \mathrm{~min}(40 \mathrm{kHz}, 100 \mathrm{~W})$. $\mathrm{GO}$ at working concentrations was prepared by diluting the stock solution with $\mathrm{K}$ medium, followed by further sonication for $30 \mathrm{~min}(40 \mathrm{kHz}, 100 \mathrm{~W})$. GO was characterized by atomic force microscopy (AFM, SPM-9600, Shimadzu, Japan), Raman spectroscopy (Renishaw Invia Plus laser Raman spectrometer, Renishaw, UK) and zeta potential by a Nano Zetasizer using a dynamic light scattering technique (Nano ZS90, Malvern Instrument, Malvern, UK). Our previous studies have examined the surface elemental composition of specific specimen using X-ray photoelectron spectrum (XPS) and oxygen functional groups in GO using Fourier transform infrared spectroscopy (FTIR), and the prepared GO has considerable degree of oxidation due to the presence of different oxygen functional groups, and the oxygen content in GO was $25.23 \% .^{13,53}$

\subsection{C. elegans strains and culture}

The used nematode strains in this study were from Caenorhabditis Genetics Center. Nematodes were maintained on nematode growth medium (NGM) plates seeded with Escherichia coli OP50 at $20{ }^{\circ} \mathrm{C} .{ }^{22}$ Gravid nematodes were lysed with a bleaching mixture $(0.45 \mathrm{M} \mathrm{NaOH}, 2 \% \mathrm{HClO})$ in order to separate eggs from adult nematodes. The collected eggs were used to prepare age synchronous L1-larvae populations.

\subsection{Exposure and toxicity assessment}

Prolonged exposure to GO from L1-larvae to adult day-1 was performed in 12-well sterile tissue culture plates in liquid $\mathrm{K}$ medium at $20{ }^{\circ} \mathrm{C}$ in the presence of food (OP50). Endpoints of ROS and locomotion behavior were selected for the toxicity assessment of GO.

We used the endpoint of intestinal reactive oxygen species (ROS) production to reflect functional state of the primary targeted organ of intestine. ROS production was analyzed as described previously. ${ }^{54}$ After labeling with CM- $\mathrm{H}_{2}$ DCFDA $(1 \mu \mathrm{M})$ for $3 \mathrm{~h}$ in the dark, the nematodes mounted on a $2 \%$ agar pad were observed and examined at $488 \mathrm{~nm}$ of excitation wavelength and at $510 \mathrm{~nm}$ of emission filter under a laser scanning confocal microscope. Relative fluorescence intensity of intestinal ROS signals was semi-quantified in comparison to intestinal autofluorescence. Thirty nematodes were examined per treatment.

Head thrash and body bend were selected to evaluate the locomotion behavior. ${ }^{55}$ Head thrash and body bend were analyzed under a dissecting microscope as described previously. ${ }^{56}$ In C. elegans, a head thrash is defined as a change in the direction of bending at the mid body. A body bend is defined as a change in the direction of the part of the nematodes corresponding to the posterior bulb of the pharynx along the $y$ axis, assuming that nematode was traveling along the $x$ axis. Thirty nematodes were examined per treatment.

\subsection{Library preparation and Illumina HiSeq 2500 sequencing}

It was reported that prolonged exposure (from L1-larvae to young adults) to $\mathrm{GO}$ at concentrations more than $0.5 \mathrm{mg} \mathrm{L}^{-1}$ could cause toxicity on the functions of both primary targeted organs and secondary targeted organs. ${ }^{57}$ The $1 \mathrm{mg} \mathrm{L}^{-1}$ was selected as working concentration for GO exposure for Illumina HiSeq2500 sequencing. For each RNA sample, total RNAs were obtained from control or GO exposed wild-type nematodes using Trizol (Invitrogen, UK) according to manufacturer's protocol. Total genomic DNA was removed using DNase I (New England Biolabs), and RNA purity was assessed using Nanodrop2000. Total RNA was subject to ribosomal RNA depletion according to manufacturer's protocol of Ribo-Minus kit. cDNA libraries were generated using TruSeq RNA Sample Prep Kit v2 (Illumina). Each library was loaded into one lane of Illumina HiSeq2500 for $2 \times 125$ bp pair-end sequencing, followed by onboard cluster generation on a Rapid Run pair-end flow cell and subsequent 125 cycles sequencing (v3 sequencing kit) according to manufacturer's instructions (HiSeq 2500, Illumina). Three independent biological replicates were performed.

\subsection{RNA-seq data analysis and circRNAs identification}

We used FastQC (http://www.bioinformatics.babraham.ac.uk/ projects/fastqc/) to control quality of sequencing data. Filtered data were mapped to nematode genome (version Caenorhabditis_elegans.WBcel235) using BWA software. ${ }^{58}$ CIRI, an efficient tool for unbiased algorithm for circRNAs identification, was used to detect the circRNAs. ${ }^{59}$ To ensure high accuracy for the identification of circRNAs, we excluded the circRNAs derived from ribosomal RNA, and selected those circRNAs identified in $\geq 2$ junction reads. To obtain full length nucleotide sequence of all circRNAs, we compared back-spliced junction sites with nematode genome annotation (version Caenorhabditis_elegans.WBcel235.81) downloaded from Ensembl database.

Expression profiling of host genes was analyzed by RSEM software, a tool for accurate transcript quantification from RNASeq data. ${ }^{60}$ Gene expression was measured in fragments per kilobase of exon per million reads mapped (FPKM). CIRI can provide expression ratio between circRNAs and their host genes. ${ }^{59}$ Based on back-spliced junction reads, edgeR, a Bioconductor package for differential expression analysis of digital gene expression data, was further used to normalize expression level of each circRNA to identify the differentially expressed genes by pairwise comparisons. ${ }^{61}$ Threshold values of $\log \mathrm{FC} \geq$ 1 and FDR (False Discovery Rate) $<0.05$ were used to judge the significance. 
2.6. Quantitative real-time polymerase chain reaction (qRTPCR) assay

For each example, total RNA extracted using Trizol (Invitrogen, UK) according to manufacturer's protocol was reverse transcribed to synthesize cDNA for qRT-PCR analysis. cDNA was synthesized as the following procedure. Firstly, 1-2 $\mu \mathrm{g}$ RNA was diluted into $14 \mu \mathrm{L}$ RNase-free water, and then $1 \mu \mathrm{L}$ random primers $(25.25 \mathrm{M}$ ) was added and mixed well. The RNA mixture was incubated at $70^{\circ} \mathrm{C}$ for $5 \mathrm{~min}$, and immediately placed on ice to incubate for $2 \mathrm{~min}$. After that, $25 \mu \mathrm{L}$ reverse transcribe reaction system containing $5 \mu \mathrm{L}$ reaction buffer, $1.3 \mu \mathrm{L}$ dNTP (10 $\mathrm{mM}$ ), $1 \mu \mathrm{L}$ M-MLV reverse transcriptase, $0.6 \mu \mathrm{L}$ RNase inhibitor, $2.1 \mu \mathrm{L}$ RNase-free water, and $15 \mu \mathrm{L}$ RNA mixture was used for real-time PCR using SYBR Premix EX TaqTM (Takara). $t b a-1$ encoding a Tubulin was used as a reference gene. Divergent and convergent primers were designed to detect the circRNAs and confirm the head-to-tail backsplicing in the circRNAs. Convergent primers were used to detect the expression of linear fragments before the head-to-tail backsplicing of circRNAs. Divergent primers were used to detect the expression of circRNAs. All circRNAs, host genes, targeted genes, and reference gene were amplified in three independent experiments. The relative level of each circRNA or targeted gene was calculated using $2^{-\Delta \Delta C_{t}}$ method. The related primer information for qRT-PCR is shown in Table S1. $\dagger$

\subsection{RNA interference (RNAi) knockdown assay}

We fed the nematodes with RNAi knockdown strains as described. ${ }^{62}$ RNAi knockdown strains grown in LB containing ampicillin $\left(100 \mu \mathrm{g} \mathrm{mL} \mathrm{m}^{-1}\right)$ at $37{ }^{\circ} \mathrm{C}$ overnight were plated onto NGM plates containing ampicillin $\left(100 \mu \mathrm{g} \mathrm{mL}^{-1}\right)$ and isopropyl 1-thio- $\beta$-D-galactopyranoside (IPTG, $5 \mathrm{mM}$ ). L1 larvae were placed on RNAi knockdown plates for 2 days until the nematodes became the gravid. The gravid adults were transferred onto fresh RNAi-expressing bacterial lawns to let them lay eggs so as to obtain the second generation of RNAi population. The RNAi efficiency was confirmed by qRT-PCR. The primer information for RNAi is shown in Table S2. $\dagger$

\subsection{Biotinylated probe of circ_oooo115}

The complementary sequence of 16 bases on each side of the back-splicing in circ_0000115 was labeled by biotin at the $5^{\prime}$ end. The sequence of circ_0000115 biotinylated probe is $5^{\prime} \mathrm{B}$ ACCGCAGCGGCCCGTTCAATTTTTTGGAATCC. biotinylation.

\section{9. circRNA pull-down}

Wild-type nematodes were harvest and washed by M9 buffer, lysed using tissue-lyser. The extract was incubated with $10 \mu \mathrm{g}$ biotinylated DNA probe against back-splicing sites of circ_0000115 in lysis buffer (20 mM HEPES (pH 7.4), $12 \mathrm{mM}$ $\mathrm{MgCl}_{2}, 200 \mathrm{mM} \mathrm{NaCl}, 1 \mathrm{mM}$ DTT, 0.1\% Triton X-100, 10\% glycerol, RNase inhibitor and protease inhibitor) at room temperature for $3 \mathrm{~h}$. A total of $50 \mu \mathrm{L}$ Dynabeads M-280 Streptavidin was added to each binding reaction, and incubated for
$2 \mathrm{~h}$ at room temperature. After washing for 3 times with lysis buffer, the samples were boiled in $6 \times$ SDS loading buffer, and subjected to SDS-PAGE gel electrophoresis. The potential protein bands were analyzed by Mass Spectrometry.

\subsection{Protein-RNA interaction prediction}

PRIdictor (Protein-RNA interaction predictor) predicts mutual binding sites in RNA and protein at the nucleotide- and residuelevel resolutions from their sequences. ${ }^{63}$ PRIdictor can be used as a web-based application or web service at http:// bclab.inha.ac.kr/pridictor.

\subsection{DNA construction and transformation}

Intestine-specific promoter Pges-1 was amplified by PCR from genomic DNA of wild-type nematodes. PCR amplified ifc-2 cDNA was inserted into vector pPD_95_77 carrying Pges-1 promoter. Germline transformation was conducted by coinjecting a testing DNA $\left(40 \mu \mathrm{g} \mathrm{mL}^{-1}\right)$ and a marker DNA of Pdop-1::rfp (60 $\mu \mathrm{g} \mathrm{mL}^{-1}$ ) into the gonad. ${ }^{64}$ Primer information for vector construction is shown in Table S3. $\dagger$

\subsection{Statistical analysis}

Data in this article were expressed as means \pm standard deviation (SD). Statistical analysis was performed using SPSS 12.0 software (SPSS Inc., Chicago, USA). Differences between groups were determined using analysis of variance (ANOVA), and probability levels of 0.05 and 0.01 were considered statistically significant. Graphs were generated using GraphPad Prism 5.0 (GraphPad Software, La Jolla, CA).

\section{Results}

\subsection{Physicochemical properties of prepared GO}

Based on the AFM assay, GO thickness was approximately $1.0 \mathrm{~nm}$, which implied the one layer property for the prepared GO (Fig. S1a†). Raman spectroscopy assay using $632 \mathrm{~nm}$ wavelength excitation indicated that GO had a D band $\left(1339.27 \mathrm{~cm}^{-1}\right)$ and a $\mathrm{G}$ band $\left(1589.5 \mathrm{~cm}^{-1}\right)$, respectively (Fig.$\mathrm{S} 1 \mathrm{~b} \dagger)$. The D-band signal appeared after treatment with sulfuric acid and $\mathrm{KMnO}_{4}$, suggesting the introduction of disorder into the graphite layer (Fig. S1b $\dagger$ ). The sizes of most of the GO after sonication were in the range of $40-50 \mathrm{~nm}$ (Fig. S1c $\dagger$ ). Zeta potential of GO in $\mathrm{K}$ medium was $-22.5 \pm 2.8 \mathrm{mV}$.

\section{2. circRNA expression profiling in GO exposed wild-type nematodes}

To systematically identify circRNAs involved in the control of response of nematodes to GO exposure, we performed the Illumina HiSeq2500 sequencing for the control group and the GO $\left(1 \mathrm{mg} \mathrm{L}^{-1}\right)$ exposure group with three duplicates. GO exposure was performed from L1-larvae to adult day-1 in wild-type N2 nematodes. A total of 339 circRNAs were detected based on the Illumina RNA-seq approach. Length distribution, chromosome distribution and genome region distribution of the circRNAs in nematodes were shown in Fig. S2. $\dagger$ Most of the 
detected circRNAs were in the range of 200-400 bp or more than $1000 \mathrm{bp}$ (Fig. S2a $\dagger$ ). Most of the detected circRNAs were on the chromosomes of I and III, and only a limited number of circRNAs were distributed on chromosome X (Fig. S2b $\dagger$ ). circRNA category shows that most of the detected circRNAs originate from the exons in nematodes (Fig. S2c $\dagger$ ).

Among these 339 circRNAs, 43 circRNAs were significantly dysregulated by GO exposure $(\log \mathrm{FC} \geq 1$, FDR $<0.05)$ (Tables $\mathrm{S} 4$ and $55 \dagger$ ). The 43 differentially expressed circRNAs were further converted into a heat map to show the distinguishable circRNAs expression profiling after GO exposure (Fig. 1a). Among these dyregulated circRNAs, 33 known circRNAs (according to http:// www.circbase.org) including 31 down-regulated circRNAs and
2 up-regulated circRNAs were identified in GO exposed wildtype nematodes (Table $\mathrm{S} 4 \uparrow$ and Fig. 1b). Moreover, 10 novel circRNAs including 7 down-regulated and 3 up-regulated circRNAs were identified in GO exposed wild-type nematodes according to Memczak's report ${ }^{\mathbf{1 7}}$ (Table $55 \dagger$ and Fig. 1c). However, based on Ivanov's report, ${ }^{65} 6$ circRNAs are known among these 10 novel circRNAs (Table S5†).

\subsection{Validation of dysregulated circRNAs in GO exposed nematodes}

We next focused on 33 known circRNAs to confirm their expressions in GO exposed nematodes using qRT-PCR, and

(a)
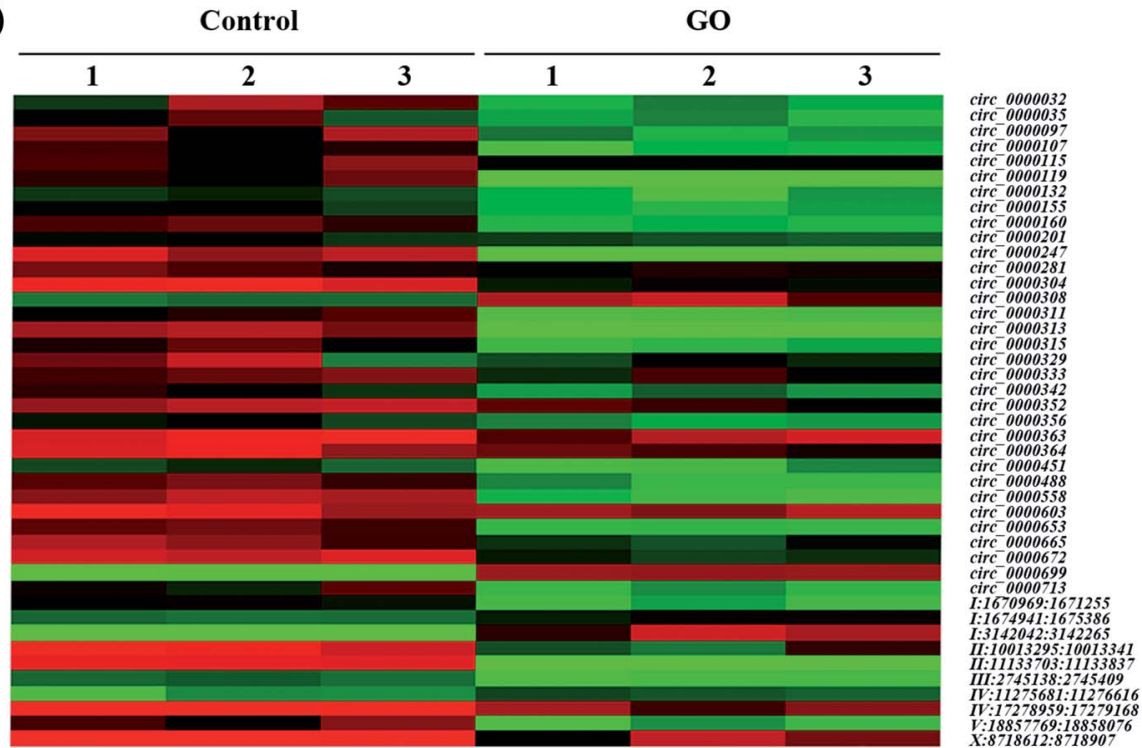

(b)

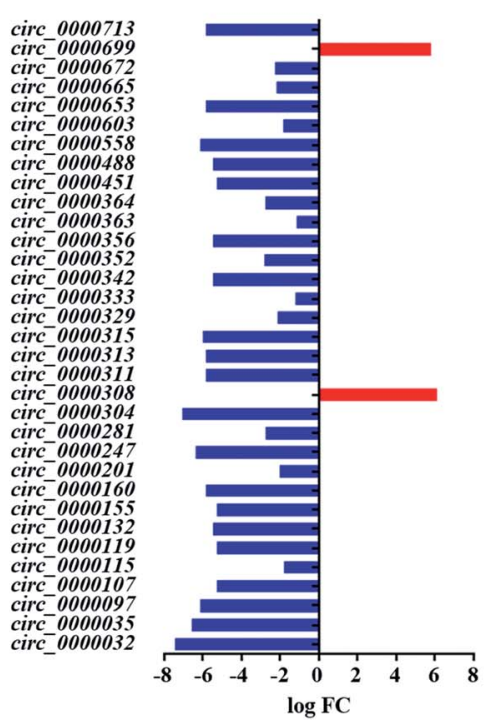

(c)
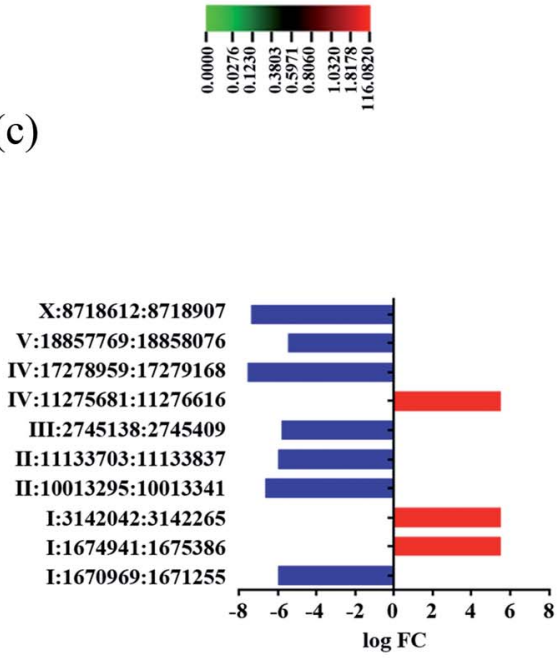

Fig. 1 Dysregulated circRNAs induced by GO exposure in wild-type nematodes. (a) Heat map of dysregulated circRNAs in GO exposed nematodes. (b) Dysregulated known circRNAs in GO exposed nematodes. (c) Dysregulated novel circRNAs in GO exposed nematodes. GO concentration is $1 \mathrm{mg} \mathrm{L}^{-1}$. Prolonged exposure to GO was performed from L1-lavae to adult day-1. 
these 33 known circRNAs are exon-shuffling-derived circRNA (Table S4 $\dagger$ ). Among the 33 known circRNAs, 28 dysregulated circRNAs were validated in GO exposed wild-type nematodes by qRT-PCR assay (Fig. 2a). Among these 28 dysregulated circRNAs, circ_000032, circ_000035, circ_000097, circ_0000107, circ_0000115, circ_0000119, circ_0000132, circ_0000160, circ_0000201, circ_0000247, circ_0000281, circ_000304, circ_0000311, circ_0000313, circ_0000329, circ_0000342, circ_0000352, circ_0000356, circ_0000363, circ_0000488, circ_0000558, circ_0000603, circ_0000653, circ_0000665, circ_0000672 and circ_0000713 were significantly downregulated in GO exposed wild-type nematodes, whereas circ_0000308 and circ_0000699 were significantly up-regulated in GO exposed wild-type nematodes (Fig. 2a). Therefore, the qRT-PCR results were largely consistent with RNA-seq data on the dysregulation of circRNAs induced by GO exposure in nematodes.

3.4. circRNAs dysregulated by GO in the range of $\mu \mathrm{g} \mathrm{L}^{-1}$ in wild-type nematodes

Considering the fact that most of the ENMs released into the

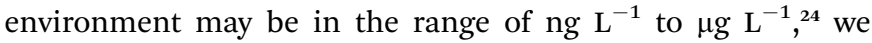
further determined the effect of GO $\left(100 \mu \mathrm{g} \mathrm{L^{-1 }}\right)$ on expression of circRNAs in nematodes (Fig. 2b). Among the 28 dysregulated circRNAs by GO $\left(1 \mathrm{mg} \mathrm{L}^{-1}\right)$, the expressions of 5 circRNAs were further significantly altered by exposure to GO $\left(100 \mu \mathrm{g} \mathrm{L}^{-1}\right)$ (Fig. 2b). These 5 dysregulated circRNAs included 4 downregulated circRNAs (circ_0000115, circ_0000201, circ_0000247 and circ_0000665) and 1 up-regulated circRNA (circ_0000308) (Fig. 2b). These five circRNAs could be further dysregulated by exposure to GO $\left(1 \mu \mathrm{g} \mathrm{L}^{-1}\right)$ from L1-larvae to adult day-8 (data not shown).

\subsection{Functional analysis of 5 circRNAs induced by GO (100} $\mu \mathrm{g} \mathrm{L}^{-1}$ )

To determine the function of these five candidate circRNAs (circ_0000115, circ_0000201, circ_0000247, circ_0000308 and circ_0000665) in regulating the GO toxicity, RNAi knockdown of these five circRNAs was performed in nematodes. The RNAi efficiency of RNAi knockdown of these five circRNAs was shown in Fig. S3. $\dagger$ Meanwhile, we found that RNAi knockdown of corresponding host genes for these five circRNAs did not affect the expressions of these five circRNAs (Fig. S3†).

To determine the function of these five candidate circRNAs in regulating the GO toxicity, ROS production and locomotion behavior were further employed as the toxicity assessment

(a)

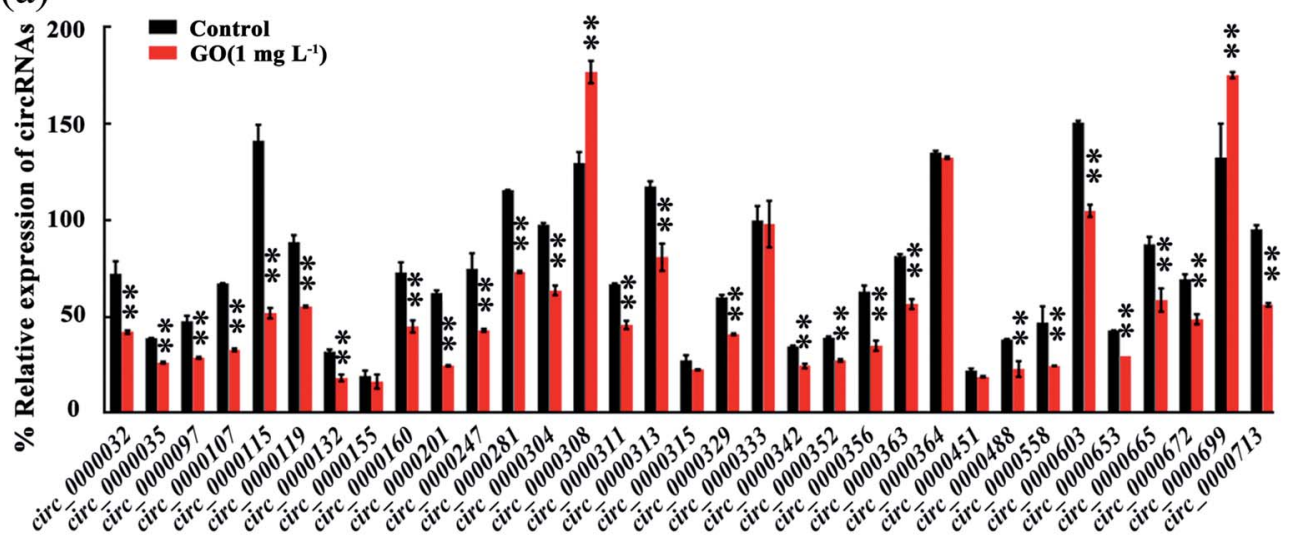

(b)

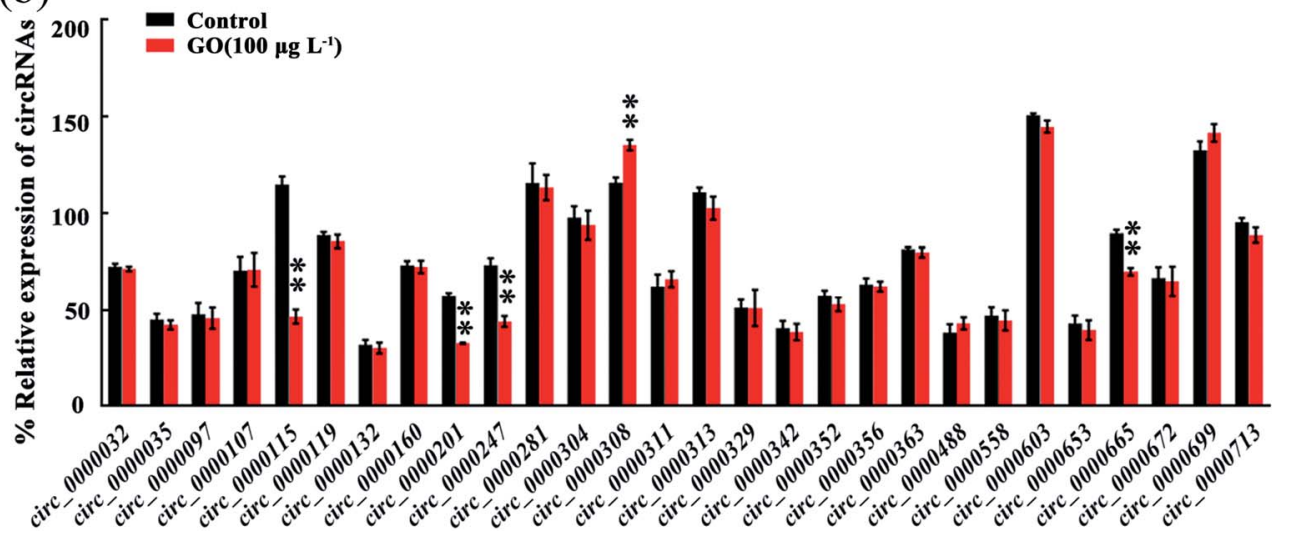

Fig. 2 Validation of circRNAs expression in GO wild-type exposed nematode via qRT-PCR analysis. (a) GO exposure concentration was 1 mg $\mathrm{L}^{-1}$. (b) GO exposure concentration was $100 \mu \mathrm{L} \mathrm{L}^{-1} \mathrm{GO}$. Prolonged exposure to GO was performed from L1-lavae to adult day-1. Bars represent means \pm SD. $* * p<0.01$ vs. control. 
endpoints. After exposure, we found that RNAi knockdown of circ_0000115, circ_0000247, circ_0000308 or circ_0000665 caused the resistance of nematodes to GO toxicity in inducing ROS production and in decreasing locomotion behavior (Fig. 3). In contrast, RNAi knockdown of circ_0000201 resulted in the susceptibility of nematodes to GO toxicity in inducing ROS production and in decreasing locomotion behavior (Fig. 3). Therefore, all these five circRNAs are involved in the regulation of GO toxicity in nematodes.

\subsection{Tissue-specific activity of circ_o000115 in the regulation of GO toxicity}

The circ_0000115 is the most down-regulated circRNA in nematodes exposed to GO, and we next focused on the circ_0000115 to examine its tissue-specific activity in regulating the GO toxicity. Using the RNAi knockdown genetic tools (VP303 used for RNAi knockdown in intestine, NR222 used for RNAi knockdown in epidermis, TU3401 used for RNAi knockdown in neurons and WM118 used for RNAi knockdown in muscle), we found that RNAi knockdown of circ_0000115 in epidermis or muscle did not affect the GO toxicity in inducing ROS production (Fig. 4). Different from this, we found that RNAi knockdown of circ_0000115 in intestine or neurons could induce a resistance to GO toxicity in inducing ROS production (Fig. 4), suggesting that the circ_0000115 may act in the intestine and the neurons to regulate the GO toxicity in nematodes.

\subsection{RNA pull-down assay to identify the targets for} circ_0000115 during the control of GO toxicity

To identify the potential targets of circ_0000115 during the control of GO toxicity, we performed a RNA pull-down experiment to pull down proteins with or without the biotinylated probe of circ_0000115. The design diagram of probe for circ_0000115 is shown in Fig. 5a. According to the results of circ_0000115 pull-down assay for the sample collected from nematodes exposure to GO $\left(100 \mu \mathrm{g} \mathrm{L}^{-1}\right)$, the amount of protein pulled down was very limited and most of the pulled down proteins were in the supernatant. Nevertheless, a visible protein band between the molecular weights of 40 to $55 \mathrm{KD}$ appeared in the sample with the biotinylated probe of circ_0000115, and this band was not present in the control without the probe (Fig. 5b). We analyzed the proteins in this significantly different band by mass spectrometry technique. The data in Table S6† lists the 15 top proteins based on the abundance of the proteins in mass spectrometry.

The RPIseq website was used to individually predict the potential binding capacity of circ_0000115 and these 15 top proteins detected by mass spectrometry. The prediction results of circ_0000115-protein binding possibility are shown in Table S7. $\dagger$ According to the score of circ_0000115-protein binding capacity, the score of mass spectrometry and phenotype of RNAi knockdown, IFC-2 protein ranked first as a possible target protein of circ_0000115 (Tables S6 and S7†). Potential nucleotide binding sites in IFC-2 amino acid sequence and potential amino acid binding sites in circ_0000115 sequence were
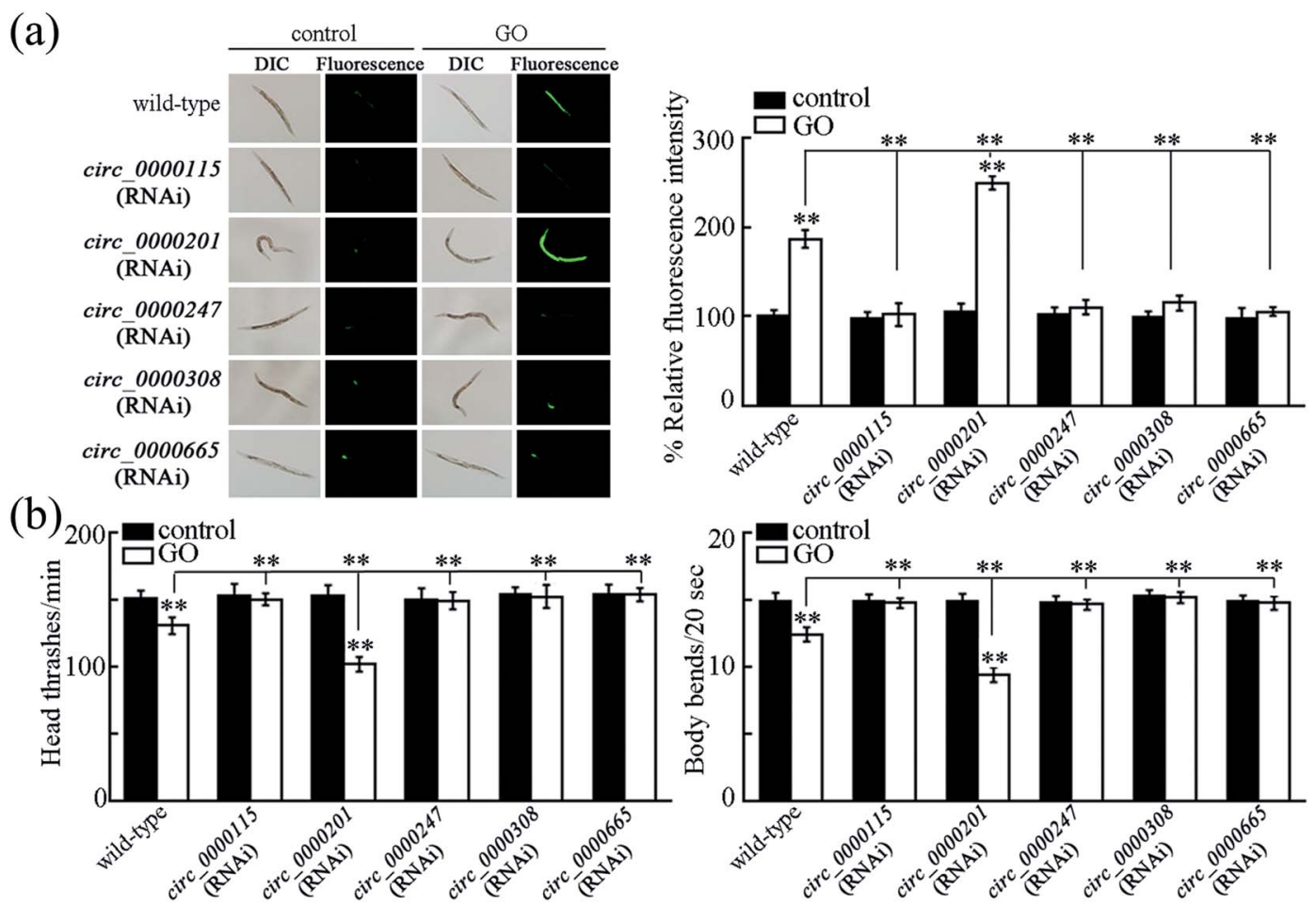

Fig. 3 Functional analysis of candidate circRNAs in regulating ROS production (a) and locomotion behavior (b). GO concentration is $100 \mu \mathrm{g} \mathrm{L}^{-1}$. Prolonged exposure to GO was performed from L1-lavae to adult day-1. Bars represent means \pm SD. **p $<0.01$ vs. control (if not specially indicated). 

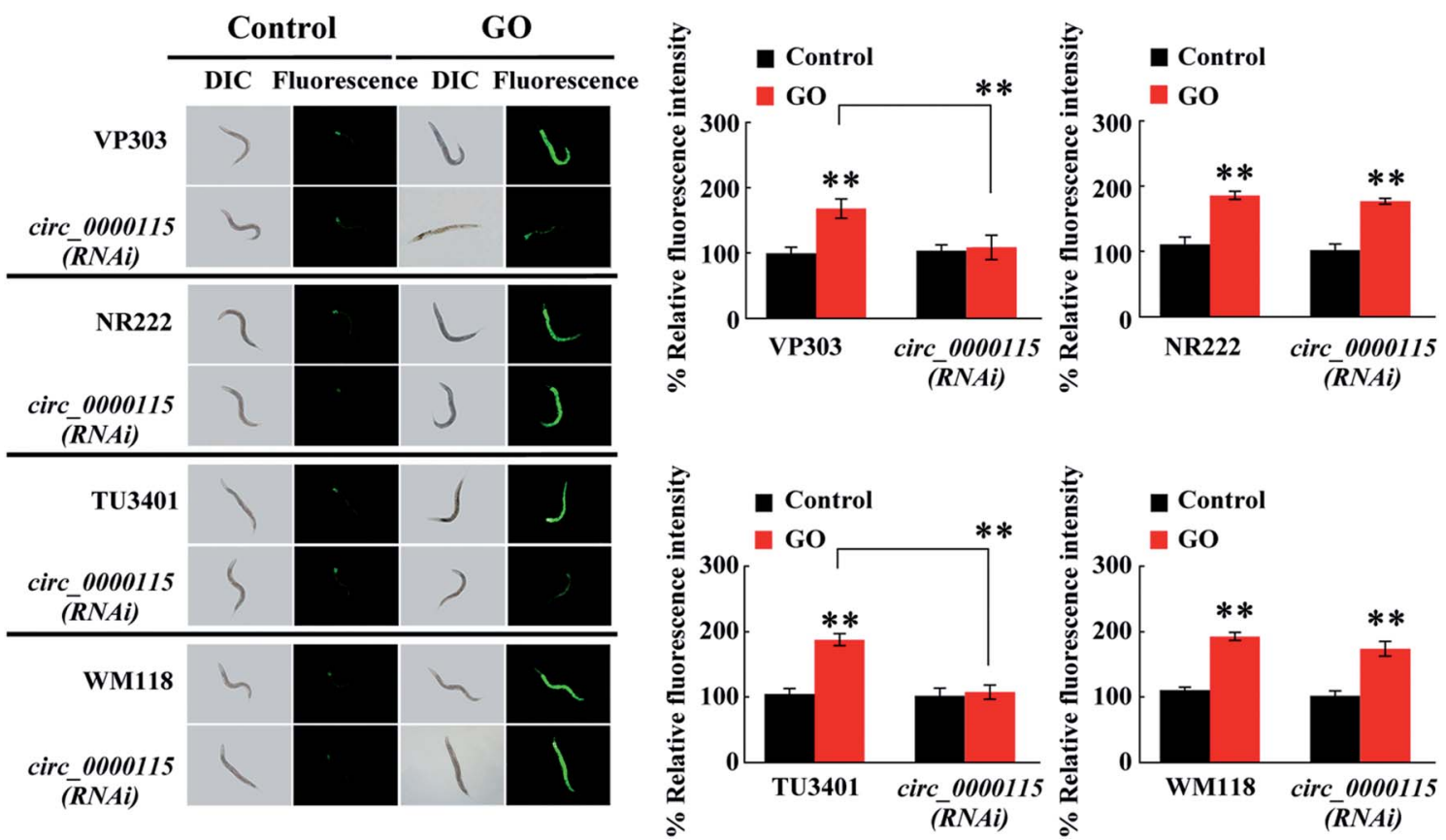

Fig. 4 Tissue-specific activity of circ_0000115 in regulating GO toxicity in inducing ROS production. GO concentration is $100 \mu \mathrm{g} \mathrm{L}^{-1}$. Prolonged exposure to GO was performed from L1-lavae to adult day-1. Bars represent means \pm SD. $* * p<0.01$ vs. control (if not specially indicated).

(a)

(c)

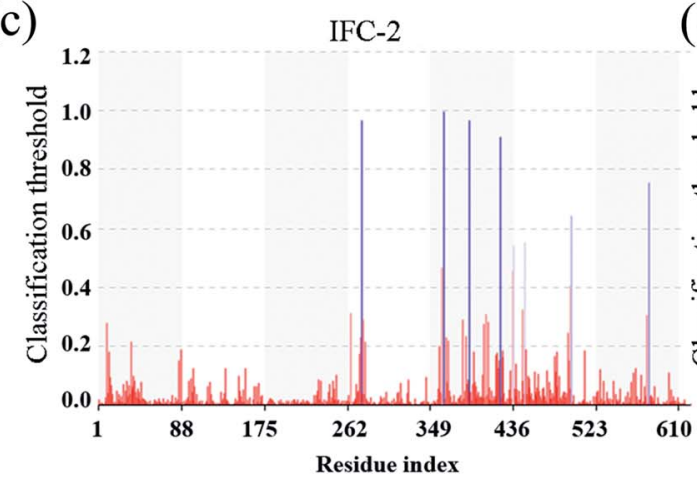

(b)

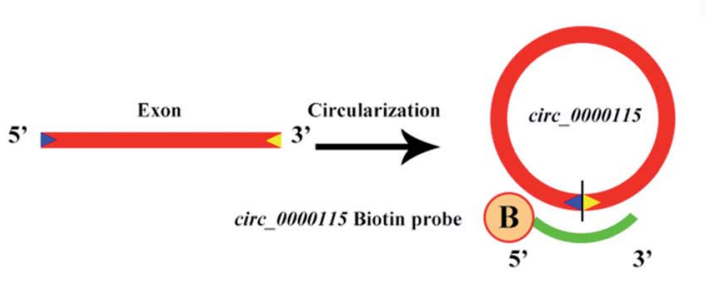

(d)
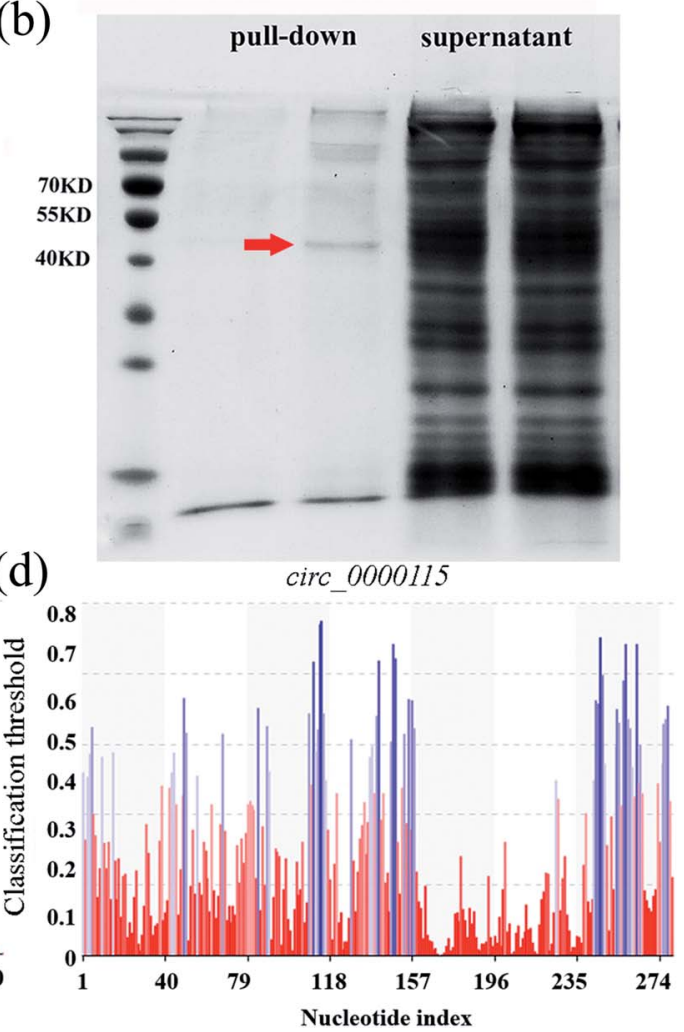

Fig. 5 circRNA pull-down assay combined with mass spectrometry analysis. (a) Diagram showing the preparation of circ_0000115 pull-down biotin probe. B: biotinylation. (b) The result of circ_0000115 pull-down in SDS-PAGE electrophoresis. Arrowhead indicates the position for IFC-2. (c) PRIdictor prediction result of the possible nucleotide binding sites in IFC-2. (d) PRIdictor prediction result of the possible amino acid binding sites in circ_0000115. 
analyzed by PRIdictor (Fig. 5c and d). The IFC-2 protein contains 8 possible nucleotide binding sites located at amino acids 276, 363, 389, 422, 436, 448, 497 and 578, respectively (Fig. 5c). circ_0000115 contains multiple sites that may bind amino acids (Fig. 5d). These results suggest the molecular interaction between circ_0000115 and IFC-2 protein in nematodes.

\subsection{Role of IFC-2 in the regulation of GO toxicity in nematodes}

In nematodes, IFC-2 is present in the cytoplasm of intestinal cells, and plays an important role in maintaining the intestinal morphological structure. ${ }^{66}$ In nematodes, exposure to GO (100 $\mu \mathrm{g} \mathrm{L}^{-1}$ ) significantly decreased the ifc-2 expression in wild-type nematodes (Fig. S4a $\dagger$ ). Meanwhile, after GO exposure, intestinespecific RNAi knockdown of circ_0000115 noticeably increased the ifc-2 expression (Fig. $\mathrm{S} 4 \mathrm{~b} \dagger$ ).

To further validate the essential roles of IFC-2 in regulating the GO toxicity, we performed the intestinal RNAi knockdown of ifc-2 in nematodes. The RNAi efficiency of ifc- 2 was confirmed by qRT-PCR (data not shown). The intestinal lumen of ifc-2 (RNAi) nematodes was considerably widened (Fig. 6a). Moreover, after GO exposure, intestinal RNAi knockdown of ifc-2 induced a more significant change of irregularly widened intestinal lumen (Fig. 6a). In nematodes, intestine-specific RNAi knockdown of ifc-2 did not affect both the intestinal permeability and the defecation behavior (Fig. S5†).

Moreover, intestinal RNAi knockdown of ifc-2 caused the more significant induction of ROS production in GO exposed nematodes compared with that in GO exposed VP303 nematodes (Fig. 6b), suggesting the formation of a susceptibility of ifc-2(RNAi) nematodes to the GO toxicity in nematodes.

\subsection{Genetic interactions between circ_0000115 and IFC-2 in regulating GO toxicity}

In nematodes, we observed that the induction of ROS production in GO exposed circ_ooo0115(RNAi); ifc-2(RNAi) nematodes was similar to that in GO exposed ifc-2(RNAi) nematodes (Fig. 6c). That is, both of these two strains showed the susceptibility to GO toxicity in inducing ROS production (Fig. 6c), suggesting that IFC-2 functions downstream of circ_0000115 to regulate the GO toxicity in nematodes.

(a)

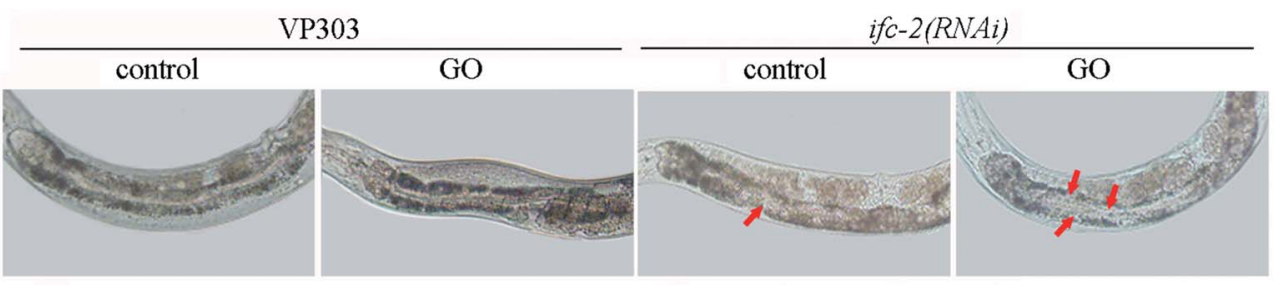

(b)

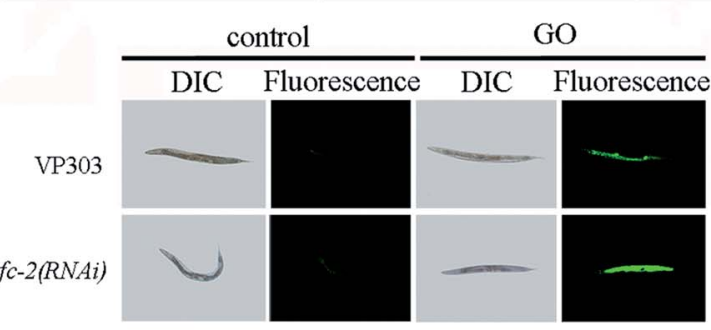

(c)
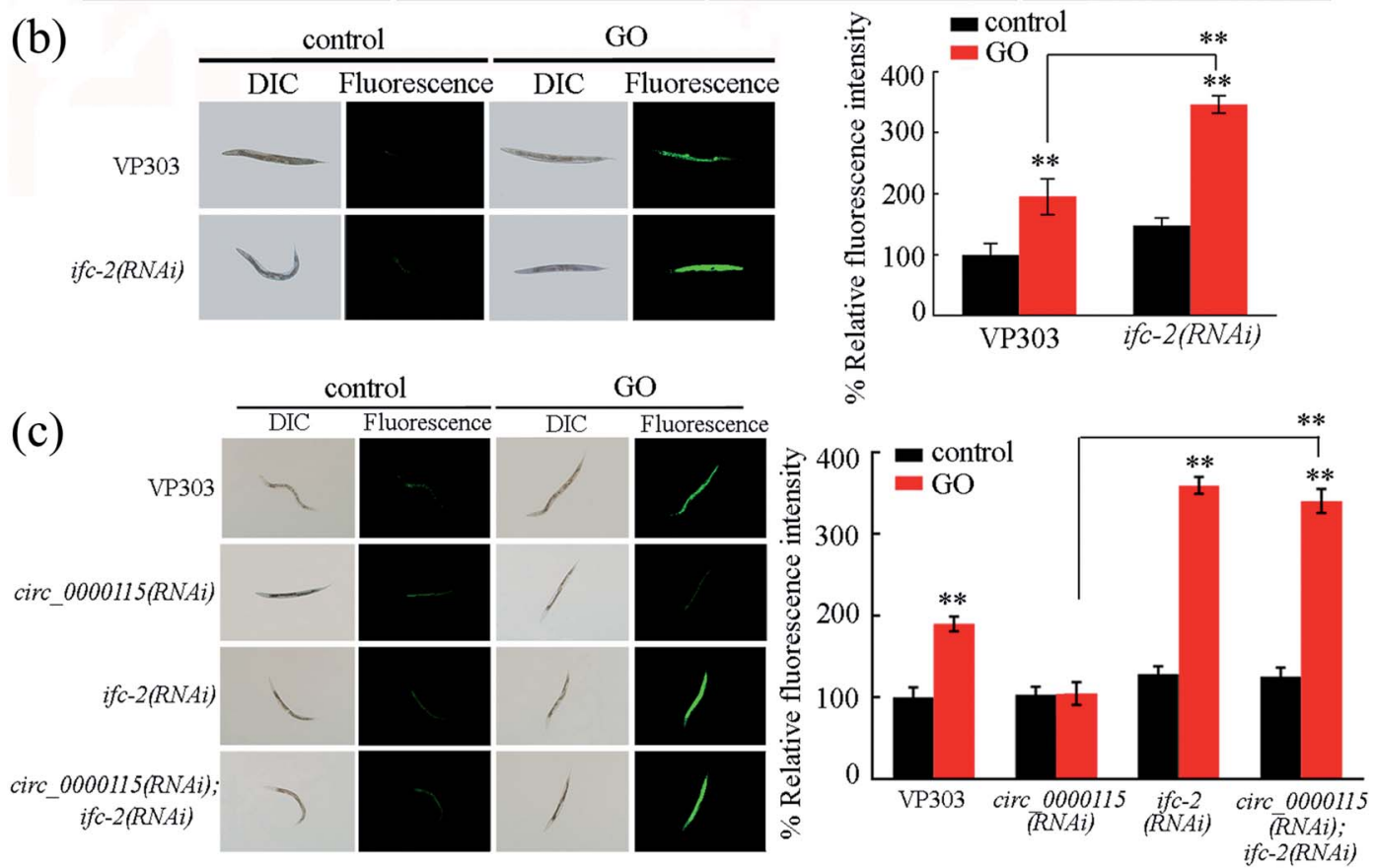

Fig. 6 Genetic interactions between circ_0000115 and IFC-2 in regulating the GO toxicity. (a) Intestinal morphology in nematodes with ifC-2 RNAi knockdown. Arrowheads indicate the altered intestinal lumen. (b) Effect of intestinal RNAi knockdown of ifc-2 on GO toxicity in inducing ROS production. (c) Genetic interactions between circ_0000115 and IFC-2 in regulating GO toxicity in inducing ROS production. GO concentration is $100 \mu \mathrm{g} \mathrm{L}^{-1}$. Prolonged exposure to GO was performed from L1-lavae to adult day-1. Bars represent means $\pm \mathrm{SD}$. $* * p<0.01 \mathrm{vs}$. control (if not specially indicated). 

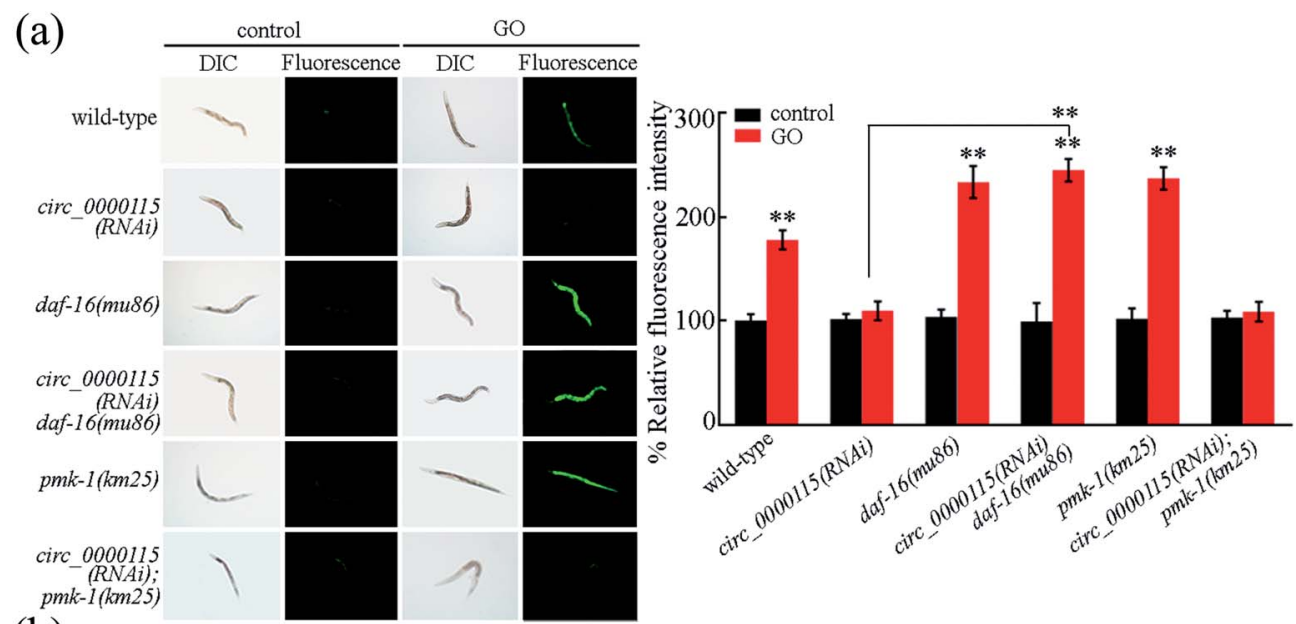

(b)
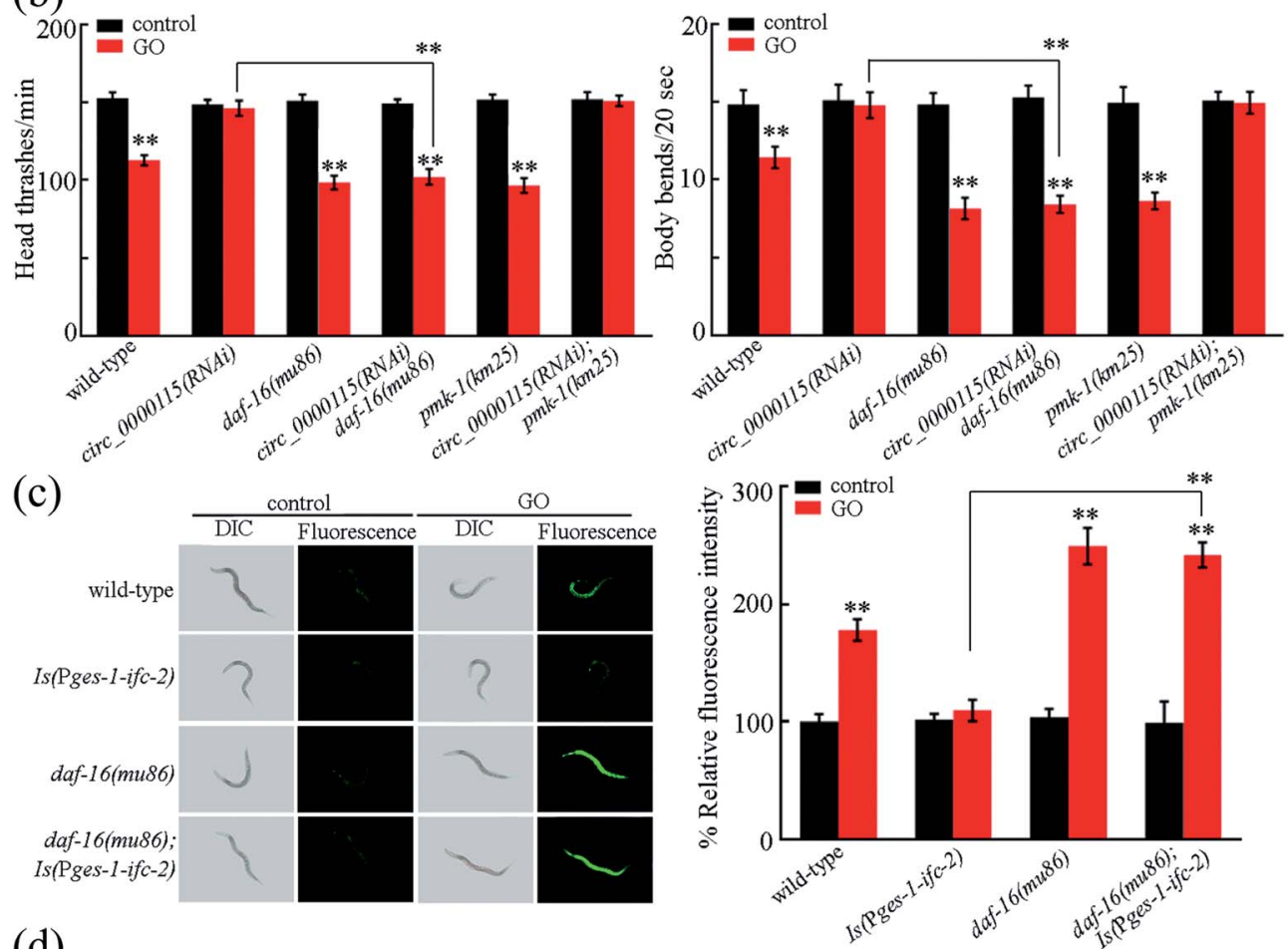

(d)
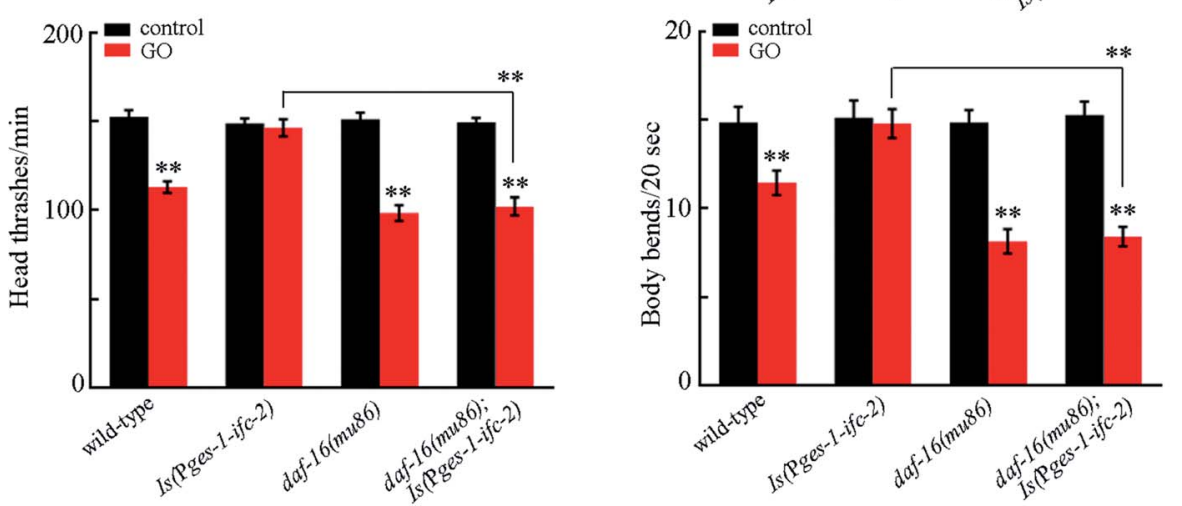

Fig. 7 IFC-2 acts upstream of DAF-16 in the insulin signaling pathway to regulate the GO toxicity. (a) Genetic interaction between circ_0000115 and DAF-16 or PMK-1 in regulating the GO toxicity in inducing ROS production. (b) Genetic interaction between circ_0000115 and DAF-16 or PMK-1 in regulating the GO toxicity in decreasing locomotion behavior. (c) Genetic interaction between IFC-2 and DAF-16 in regulating the GO toxicity in inducing ROS production. (d) Genetic interaction between IFC-2 and DAF-16 in regulating the GO toxicity in decreasing locomotion behavior. GO concentration is $100 \mu \mathrm{g} \mathrm{L}^{-1}$. Prolonged exposure to $\mathrm{GO}$ was performed from L1-lavae to adult day-1. Bars represent means \pm SD. $* * p<0.01$ vs. control (if not specially indicated). 
3.10. DAF-16 in the insulin signaling pathway acts as a downstream target of intestinal IFC-2 in the regulation of GO toxicity

In nematodes, insulin signaling and p38 MAPK signaling act in the intestine to regulate the GO toxicity. ${ }^{24,47,49}$ In the insulin signaling pathway, daf-16 encodes a FOXO transcriptional factor. In the p38 MAPK signaling pathway, $p m k-1$ encodes a p38 MAPK. We found that mutation of $d a f-16$, but not the mutation of pmk-1, could suppress the resistance of circ_0000115(RNAi) nematodes to the GO toxicity in inducing ROS production and in decreasing locomotion behavior (Fig. 7a and b). Therefore, the circ_0000115 regulates the GO toxicity by acting upstream of insulin signaling in nematodes.

In nematodes, intestinal overexpression of IFC-2 could suppress the induction of ROS production and the decrease in locomotion behavior in GO exposed nematodes (Fig. 7c and d), suggesting that the intestinal overexpression of IFC-2 may induce a resistance to GO toxicity. Moreover, we observed that daf-16 mutation could further inhibit the resistance of nematodes overexpressing intestinal IFC-2 to the GO toxicity in inducing ROS production and in decreasing locomotion behavior (Fig. 7c and d). Therefore, DAF-16 in the insulin signaling pathway can further act downstream of IFC-2 to regulate the $\mathrm{GO}$ toxicity in nematodes.

\section{Discussion}

In organisms, previous studies have demonstrated that miRNAs could be involved in the control of response to GO exposure. ${ }^{\mathbf{1 0 , 1 4}}$ More recently, it was further reported that some lncRNAs such as linc-37 also participated in the regulation of response of organisms to GO exposure. ${ }^{13}$ So far, an accumulating evidence has indicated that the circRNAs are not simply the by-products of mis-splicing or splicing errors, and some circRNAs have been proven to play important functions during the development of organisms. ${ }^{67-69}$ In this study, our data demonstrate that five circRNAs were also associated with the induction of GO toxicity in nematodes. Based on the Illumina HiSeq2500 sequencing, we identified 43 dysregulated circRNAs among the detected 339 circRNAs in GO $\left(1 \mathrm{mg} \mathrm{L}^{-1}\right)$ exposed wild-type nematodes (Tables S4 and S5 $\dagger$ ). Among these 43 dysregulated circRNAs in GO (1 $\mathrm{mg} \mathrm{L}^{-1}$ ) exposed wild-type nematodes, 38 circRNAs were down-regulated, and 5 circRNAs were up-regulated (Tables S4 and S5, $\uparrow$ Fig. 1). Moreover, 5 circRNAs could be further dysregulated by exposure to GO in the range of $\mu \mathrm{g} \mathrm{L}^{-1}$ (Fig. 2), implying that environmental exposure to GO in the range of $\mu \mathrm{g}$ $\mathrm{L}^{-1}$ may affect the expressions of these circRNAs. Therefore, our results imply that a limited number of circRNAs may be associated with the toxicity formation in nematodes exposed to GO in the range of $\mu \mathrm{g} \mathrm{L}^{-1}$. In this study, we also identified 136 novel circRNAs, and these identified novel circRNAs provide an important basis for systematic analysis of the structures and the functions of circRNAs in the regulation of various biological processes in nematodes.

In order to determine the functions of candidate circRNAs in the regulation of GO toxicity, we performed the RNAi knockdown experiments. The specific RNAi knockdown efficiency of candidate circRNAs was confirmed by using two types of targeted RNAis (circRNA RNAi and host gene RNAi) (Fig. S3 $\dagger$ ). After GO exposure, we found that RNAi knockdown of four candidate circRNAs (circ_0000115, circ_0000247, circ_0000308 and circ_0000665) caused a resistance to GO toxicity, and RNAi knockdown of one candidate circRNA (circ_0000201) resulted in a susceptibility to GO toxicity (Fig. 3). Meanwhile, GO exposure decreased the expressions of circ_0000115, circ_0000201, circ_0000247 and circ_0000665, and increased the expressions of circ_0000308 (Fig. 2b). Therefore, the alteration in expressions of circ_0000115, circ_0000247 and circ_0000665 may mediate a protective response for nematodes to GO exposure, whereas the alteration in expressions of circ_0000201 and circ_0000308 may mediate the toxicity induction of GO in nematodes. That is, the altered circRNAs mediate two different responses for nematodes to GO exposure.

Previous studies have highlighted that GO can be distributed and accumulated into various organs, including the primary targeted organs (such as intestine) and the secondary targeted organs (such as neurons and reproductive organs). ${ }^{24}$ With the aid of certain tissue RNAi knockdown genetic tools, we found that circ_0000115 might mainly play a role in regulating the GO toxicity in two tissues including the intestine and the neurons (Fig. 4). These observations imply that circ_0000115 mediated certain signaling pathways in the intestine and the neurons, respectively, to regulate the GO toxicity in nematodes.

We further focused on the circ_0000115 to examine the underlying molecular mechanism of circRNAs in the regulation of GO toxicity. The results of RNA pull-down experiments and PRIdictor analysis implied that there was an interaction between circ_0000115 and IFC-2 protein (Fig. 5). Genetic interaction analysis confirmed that IFC-2 acted downstream of circ_0000115 to regulate the GO toxicity (Fig. 6c). In C. elegans, ifc-2 encodes three isoforms of an intermediate filament protein dispensable for viability but required for intestinal tube maintenance. ${ }^{66}$ Therefore, RNAi knockdown of intestinal circ_0000115 may prevent the GO toxicity by suppressing the damage of ifc-2 deficit in the intestine.

In nematodes, GO exposure suppressed the IFC-2 expression (Fig. S4a†). Meanwhile, RNAi knockdown of ifc-2 induced the severe intestinal morphology changes of the irregular widen lumen and the intestinal ROS production in nematodes exposed to GO (Fig. 6a and b). Therefore, the expressional alteration of IFC-2 acts as an important molecular basis for GO toxicity induction, and this molecular basis can be inhibited by the decrease in circ_0000115 expression in GO exposed nematodes. In nematodes, although intestinal RNAi knockdown of ifc-2 did not affect the intestinal permeability, intestinal RNAi knockdown of ifc-2 enlarged the intestinal lumen (Fig. S5a $\dagger$ ). Meanwhile, the ifc-2(RNAi) nematodes had the normal defecation behavior (Fig. S5b $\dagger$ ). These observations imply that the detected susceptibility to GO toxicity in ifc-2(RNAi) nematodes may be largely due to the enlargement of intestinal lumen and the more severe accumulation of GO in intestinal lumen (data not shown). 
In this study, we also examined the role of other possible targets of circ_0000115 based on the RNA pull-down assay. Since clik-1, hsp-43 and car-1 are not expressed in the intestine or the neuron, we did not examine them. Among the rest genes, RNAi knockdown of nep-17, eft-3 or vit-6 resulted in a moderate susceptibility of nematodes to the GO toxicity in inducing intestinal ROS production, and RNAi knockdown of hrpk-1 showed a moderate resistance to the GO toxicity in inducing intestinal ROS production (Fig. S6†). Therefore, besides IFC-2, NEP-17, EFT-3, VIT-6, and HRPK-1 may be also able to act as downstream targets of circ_0000115 in the regulation of GO toxicity in nematodes.

In nematodes, some molecular signaling pathways such as insulin signaling and p38 MAPK signaling pathways acted in the intestine to regulate the GO toxicity. ${ }^{24}$ Nevertheless, genetic interaction analysis suggested that p38 MAPK signaling may not act downstream of circ_0000115 to regulate the GO toxicity (Fig. 7a and b). Different from this, circ_0000115 acted upstream of the insulin signaling to regulate the GO toxicity (Fig. 7a and b). Moreover, the evidence was provided to show that the FOXO transcriptional factor DAF-16 in the insulin signaling pathway further acted downstream of intestinal IFC-2 to regulate the GO toxicity (Fig. 7c and d). Therefore, an intestinal signaling cascade of circ_0000115-IFC-2-DAF-16 was raised to be required for the regulation of GO toxicity in nematodes (Fig. 8). In nematodes, the daf-16 mutants do not show the obvious deficits in the intestine, which suggests that other

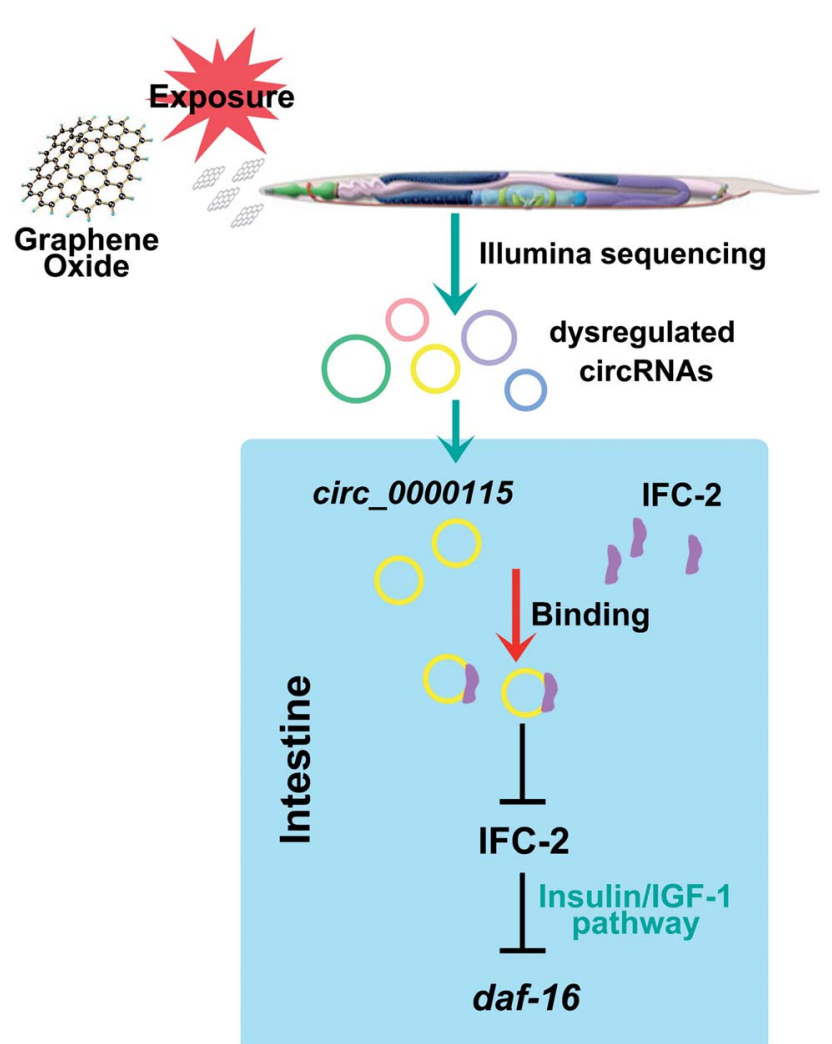

Fig. 8 A diagram showing the intestinal circ_0000115-IFC-2-DAF-16 signaling cascade involved in the control of GO toxicity in nematodes. important downstream targets for intestinal IFC-2 still remain unclear. Additionally, the underlying mechanism for neuronal circ_0000115 in the regulation of GO toxicity still needs to the further elucidation.

\section{Conclusions}

In this study, we first employed the model animal of C. elegans as the assay system to identify the potential dysregulated circRNAs induced by GO exposure. We have identified 43 dysregulated circRNAs among the detected 339 circRNAs in GO $\left(1 \mathrm{mg} \mathrm{\textrm {L } ^ { - 1 }}\right.$ ) exposed nematodes. Five dysregulated circRNAs could be further dysregulated by GO exposure in the range of $\mu \mathrm{g}$ $\mathrm{L}^{-1}$. Using the RNAi knockdown technique, we found that the candidate five circRNAs mediated two different responses for nematodes to GO exposure. With the circ_0000115 as an example, we found that circ_0000115 acted in both the intestine and the neurons to regulate the GO toxicity. For the underlying molecular mechanism for circ_0000115 in the regulation of GO toxicity, the IFC-2 was identified as the target of circ_0000115. Moreover, an intestinal signaling cascade of circ_0000115-IFC-2DAF-16 has been raised to be required for the control of GO toxicity in nematodes. Our study identified the mechanism for intestinal circ_0000115 in response to GO in organisms.

\section{Conflicts of interest}

There are no conflicts to declare.

\section{Acknowledgements}

This work was supported by the grant from National Natural Science Foundation of China (21577016).

\section{References}

1 A. K. Geim, Graphene: status and prospects, Science, 2009, 324, 1530-1534.

2 A. K. Geim and K. S. Novoselov, The rise of grapheme, Nat. Mater., 2007, 6, 183-191.

3 H. Y. Mao, S. Laurent, W. Chen, O. Akhavan, M. Imani, A. A. Ashkarran, et al., Graphene: promises, facts, opportunities, and challenges in nanomedicine, Chem. Rev., 2013, 113, 3407-3424.

4 D. Bitounis, H. Ali-Boucetta, B. H. Hong, D. H. Min and K. Kostarelos, Prospects and challenges of graphene in biomedical applications, Adv. Mater., 2013, 25, 2258-2268.

5 O. Akhavan, E. Ghaderi, S. A. Shirazian and R. Rahighi, Rolled graphene oxide foams as three-dimensional scaffolds for growth of neural fibers using electrical stimulation of stem cells, Carbon, 2016, 97, 71-77.

6 F. Perreault, H. Jaramillo, M. Xie, M. Ude, L. D. Nghiem and M. Elimelech, Biofouling mitigation in forward osmosis using graphene oxide functionalized thin-film composite membranes, Environ. Sci. Technol., 2016, 50, 5840-5848.

7 H. N. Nguyen, S. L. Castro-Wallace and D. F. Rodrigues, Acute toxicity of graphene nanoplatelets on biological 
wastewater treatment process, Environ. Sci.: Nano, 2017, 4, 160-169.

8 K. Yang, Y. Li, X. Tan, R. Peng and Z. Liu, Behavior and toxicity of graphene and its functionalized derivatives in biological systems, Small, 2013, 9, 1492-1503.

9 J. P. Souza, J. F. Baretta, F. Santos, I. M. M. Paino and V. Zucolotto, Toxicological effects of graphene oxide on adult zebrafish (Danio rerio), Aquat. Toxicol., 2017, 186, 1118.

10 Y.-P. Li, Q.-L. Wu, Y.-L. Zhao, Y.-F. Bai, P.-S. Chen, T. Xia, et al., Response of microRNAs to in vitro treatment with graphene oxide, ACS Nano, 2014, 8, 2100-2110.

11 M. Dziewięcka, J. Karpeta-Kaczmarek, M. Augustyniak, L. Majchrzycki and M. A. Augustyniak-Jablokow, Evaluation of in vivo graphene oxide toxicity for Acheta domesticus in relation to nanomaterial purity and time passed from the exposure, J. Hazard. Mater., 2016, 305, 30-40.

12 G. Qu, S. Liu, S. Zhang, L. Wang, X. Wang, B. Sun, et al., Graphene oxide induces Toll-like receptor 4 (TLR4)dependent necrosis in macrophages, ACS Nano, 2013, 7, 5732-5745.

13 Q.-L. Wu, X.-F. Zhou, X.-X. Han, Y.-Z. Zhuo, S.-T. Zhu, Y.-L. Zhao, et al., Genome-wide identification and functional analysis of long noncoding RNAs involved in the response to graphene oxide, Biomaterials, 2016, 102, 277-291.

14 Q.-L. Wu, Y.-L. Zhao, G. Zhao and D.-Y. Wang, microRNAs control of in vivo toxicity from graphene oxide in Caenorhabditis elegans, Nanomedicine, 2014, 10, 1401-1410.

15 L. Chen, C. Huang, X. Wang and G. Shan, Circular RNAs in eukaryotic cells, Curr. Genomics, 2015, 16, 312-318.

16 J. Salzman, R. E. Chen, M. N. Olsen, P. L. Wang and P. O. Brown, Cell-type specific features of circular RNA expression, PLoS Genet., 2013, 9, e1003777.

17 S. Memczak, M. Jens, A. Elefsinioti, F. Torti, J. Krueger, A. Rybak, et al., Circular RNAs are a large class of animal RNAs with regulatory potency, Nature, 2013, 495, 333-338.

18 J. N. Boeckel, N. Jae, A. W. Heumuller, W. Chen, R. A. Boon, K. Stellos, et al., Identification and characterization of hypoxia-regulated endothelial circular RNA, Circ. Res., 2015, 117, 884-890.

19 S. Haque and L. W. Harries, Circular RNAs (circRNAs) in Health and Disease, Gene, 2017, 8, pii: E353.

20 L. Szabo and J. Salzman, Detecting circular RNAs: bioinformatic and experimental challenges, Nat. Rev. Genet., 2016, 17, 679-692.

21 M. Qiu, W. Xia, R. Chen, S. Wang, Y. Xu, Z. Ma, et al., The circular RNA circPRKCI promotes tumor growth in lung adenocarcinoma, Cancer Res., 2018, 78, 2839-2851.

22 S. Brenner, The genetics of Caenorhabditis elegans, Genetics, 1974, 77, 71-94.

23 M. C. Leung, P. L. Williams, A. Benedetto, C. Au, K. J. Helmcke, M. Aschner, et al., Caenorhabditis elegans: an emerging model in biomedical and environmental toxicology, Toxicol. Sci., 2008, 106, 5-28.

24 D.-Y. Wang, Nanotoxicology in Caenorhabditis elegans, Springer Nature Singapore Pte Ltd., 2018.
25 V. L. Castro, Z. Clemente, C. Jonsson, M. Silva, J. H. Vallim, A. M. Z. de Medeiros and D. S. T. Martinez, Nanoecotoxicity assessment of graphene oxide and its relationship with humic acid, Environ. Toxicol. Chem., 2018, 37, 1998-2012.

26 N. Chatterjee, J. S. Yang, K. Park, S. M. Oh, J. Park and J. Choi, Screening of toxic potential of graphene family nanomaterials using in vitro and alternative in vivo toxicity testing systems, Environ Health Toxicol., 2015, 30, e2015007.

27 J. N. Meyer, C. A. Lord, X. Y. Yang, E. A. Turner, A. R. Badireddy, S. M. Marinakos, et al., Intracellular uptake and associated toxicity of silver nanoparticles in Caenorhabditis elegans, Aquat. Toxicol., 2010, 100, 140-150.

28 H. Ma, P. M. Bertsch, T. C. Glenn, N. J. Kabengi and P. L. Williams, Toxicity of manufactured zinc oxide nanoparticles in the nematode Caenorhabditis elegans, Environ. Toxicol. Chem., 2009, 28, 1324-1330.

29 M. Walczynska, W. Jakubowski, T. Wasiak, K. Kadziola, N. Bartoszek, S. Kotarba, M. Siatkowska, P. Komorowski and B. Walkowiak, Toxicity of silver nanoparticles, multiwalled carbon nanotubes, and dendrimers assessed with multicellular organism Caenorhabditis elegans, Toxicol. Mech. Methods, 2018, 28, 432-439.

30 S. Jung, X. Qu, B. Aleman-Meza, T. Wang, C. Riepe, Z. Liu, et al., Multi-endpoint, high-throughput study of nanomaterial toxicity in Caenorhabditis elegans, Environ. Sci. Technol., 2015, 49, 2477-2485.

31 Y.-L. Zhao, Q.-L. Wu, Y.-P. Li and D.-Y. Wang, Translocation, transfer, and in vivo safety evaluation of engineered nanomaterials in the non-mammalian alternative toxicity assay model of nematode Caenorhabditis elegans, RSC Adv., 2013, 3, 5741-5757.

32 S. Rogers, K. M. Rice, N. D. Manne, T. Shokuhfar, K. He, V. Selvaraj and E. R. Blough, Cerium oxide nanoparticle aggregates affect stress response and function in Caenorhabditis elegans, SAGE Open Med., 2015, 3, 2050312115575387.

33 D. L. Starnes, S. S. Lichtenberg, J. M. Unrine, C. P. Starnes, E. K. Oostveen, et al., Distinct transcriptomic responses of Caenorhabditis elegans to pristine and sulfidized silver nanoparticles, Environ. Pollut., 2016, 213, 314-321.

34 Y. J. Cha, J. Lee and S. S. Choi, Apoptosis-mediated in vivo toxicity of hydroxylated fullerene nanoparticles in soil nematode Caenorhabditis elegans, Chemosphere, 2012, 87, 49-54.

35 E. Zanni, G. De Bellis, M. P. Bracciale, A. Broggi, M. I. Santarelli, M. S. Sarto, et al., Graphite nanoplatelets and Caenorhabditis elegans: insights from an in vivo model, Nano Lett., 2012, 12, 2740-2744.

36 O. V. Tsyusko, J. M. Unrine, D. Spurgeon, E. Blalock, D. Starnes, M. Tseng, et al., Toxicogenomic responses of the model organism Caenorhabditis elegans to gold nanoparticles, Environ. Sci. Technol., 2012, 46, 4115-4124.

37 C. Fajardo, G. Costa, M. Nande, C. Martín, M. Martín and S. Sánchez-Fortún, Heavy metals immobilization capability of two iron-based nanoparticles (nZVI and $\mathrm{Fe}_{3} \mathrm{O}_{4}$ ): soil and freshwater bioassays to assess ecotoxicological impact, Sci. Total Environ., 2019, 656, 421-432. 
38 S. W. Kim, J. I. Kwak and Y. An, Multigenerationl study of gold nanoparticles in Caenorhabditis elegans: transgenerational effect of maternal exposure, Environ. Sci. Technol., 2013, 47, 5393-5399.

39 A. Scharf, A. Piechulek and A. Von Mikeca, Effect of nanoparticles on the biochemical and behavioral aging phenotype of the nematode Caenorhabditis elegans, ACS Nano, 2013, 12, 10695-10703.

40 H. J. Eom, C. P. Roca, J. Y. Roh, N. Chatterjee, J. S. Jeong, et al., A systems toxicology approach on the mechanism of uptake and toxicity of MWCNT in Caenorhabditis elegans, Chem.-Biol. Interact., 2015, 239, 153-163.

41 L. Gonzalez-Moragas, A. Roig and A. Laromaine, C. elegans as a tool for in vivo nanoparticle assessment, Adv. Colloid Interface Sci., 2015, 219, 10-26.

42 C. Huang, S. Li and V. Liao, Chronic ZnO-NPs exposure at environmentally relevant concentrations results in metabolic and locomotive toxicities in Caenorhabditis elegans, Environ. Pollut., 2017, 220, 1456-1464.

43 S. Bosch, T. L. Botha, A. Jordaan, M. Maboeta and V. Wepener, Sublethal effects of ionic and nanogold on the nematode Caenorhabditis elegans, J. Toxicol., 2018, 2018, 6218193.

44 W. Zhang, C. Wang, Z. Li, Z. Lu, Y. Li, J. Yin, et al., Unraveling stress-induced toxicity properties of graphene oxide and the underlying mechanism, Adv. Mater., 2012, 24, 5391-5397.

45 N. Chatterjee, Y. Kim, J. Yang, C. P. Roca, S. W. Joo and J. Choi, A systems toxicology approach reveals the WntMAPK crosstalk pathway mediated reproductive failure in Caenorhabditis elegans exposed to graphene oxide (GO) but not to reduced graphene oxide (rGO), Nanotoxicology, 2017, 11, 76-86.

46 D. A. Arndt, E. K. Oostveen, J. Triplett, D. A. Butterfield, O. V. Tsyusko, B. Collin, D. L. Starnes, et al., The role of charge in the toxicity of polymer-coated cerium oxide nanomaterials to Caenorhabditis elegans, Comp. Biochem. Physiol., Part C: Toxicol. Pharmacol., 2017, 201, 1-10.

47 M.-X. Ren, L. Zhao, X.-C. Ding, N. Krasteva, Q. Rui and D.-Y. Wang, Developmental basis for intestinal barrier against the toxicity of graphene oxide, Part. Fibre Toxicol., 2018, 15, 26.

48 M. J. Mashock, T. Zanon, A. D. Kappell, L. N. Petrella, E. C. Andersen and K. R. Hristova, Copper oxide nanoparticles impact several toxicological endpoints and cause neurodegeneration in Caenorhabditis elegans, PLoS One, 2016, 11, e0167613.

49 Y.-L. Zhao, L.-T. Zhi, Q.-L. Wu, Y.-L. Yu, Q.-Q. Sun and D.-Y. Wang, p38 MAPK-SKN-1/Nrf signaling cascade is required for intestinal barrier against graphene oxide toxicity in Caenorhabditis elegans, Nanotoxicology, 2016, 10, 1469-1479.

50 M.-X. Ren, L. Zhao, X. Lv and D.-Y. Wang, Antimicrobial proteins in the response to graphene oxide in Caenorhabditis elegans, Nanotoxicology, 2017, 11, 578-590.

51 M. Qu, Y.-H. Li, Q.-L. Wu, Y.-K. Xia and D.-Y. Wang, Neuronal ERK signaling in response to graphene oxide in nematode Caenorhabditis elegans, Nanotoxicology, 2017, 11, 520-533.

52 L.-T. Zhi, M. Qu, M.-X. Ren, L. Zhao, Y.-H. Li and D.-Y. Wang, Graphene oxide induces canonical Wnt/ $\beta$-catenin signalingdependent toxicity in Caenorhabditis elegans, Carbon, 2017, 113, 122-131.

53 Q.-L. Wu, Y.-L. Zhao, J.-P. Fang and D.-Y. Wang, Immune response is required for the control of in vivo translocation and chronic toxicity of graphene oxide, Nanoscale, 2014, 6, 5894-5906.

54 Q.-L. Ruan, Y. Qiao, Y.-L. Zhao, Y. Xu, M. Wang, J.-A. Duan, et al., Beneficial effects of Glycyrrhizae radix extract in preventing oxidative damage and extending the lifespan of Caenorhabditis elegans, J. Ethnopharmacol., 2016, 177, 101110.

55 E. L. Tsalik and O. Hobert, Functional mapping of neurons that control locomotory behavior in Caenorhabditis elegans, J. Neurobiol., 2003, 56, 178-197.

56 G.-S. Xiao, L. Zhao, Q. Huang, H.-H. Du, D.-Q. Guo, M.-X. Xia, et al., Biosafety assessment of water samples from Wanzhou watershed of Yangtze Three Gorges Reservoir in the quiet season in Caenorhabditis elegans, Sci. Rep., 2018, 8, 14102.

57 Q.-L. Wu, L. Yin, X. Li, M. Tang, T. Zhang and D.-Y. Wang, Contributions of altered permeability of intestinal barrier and defecation behavior to toxicity formation from graphene oxide in nematode Caenorhabditis elegans, Nanoscale, 2013, 5, 9934-9943.

$58 \mathrm{H}$. Li, Aligning sequence reads, clone sequences and assembly contigs with BWA-MEM, 2013, arXiv, 1303, 3997.

59 Y. Gao, J. Wang and F. Zhao, CIRI: an efficient and unbiased algorithm for de novo circular RNA identification, Genome Biol., 2015, 16, 4.

60 B. Li and C. N. Dewey, RSEM: accurate transcript quantification from RNA-seq data with or without a reference genome, BMC Bioinf., 2011, 12, 323.

61 M. D. Robinson, D. J. McCarthy and G. K. Smyth, edgeR: a bioconductor package for differential expression analysis of digital gene expression data, Bioinformatics, 2010, 26, 139-140.

62 A. Sinha and R. Rae, Genome-Wide RNAi Screens in $C$. elegans to identify genes influencing lifespan and innate immunity, Methods Mol. Biol., 2016, 1470, 171-182.

63 U. M. Muppirala, B. A. Lewis and D. Dobbs, Computational tools for investigating RNA-protein interaction partners, $J$. Comput. Sci. Syst. Biol., 2013, 6, 182-187.

64 C. Mello and A. Fire, DNA transformation, Methods Cell Biol., 1995, 48, 451-482.

65 A. Ivanov, S. Memczak, E. Wyler, F. Torti, H. T. Porath, M. R. Orejuela, et al., Analysis of intron sequences reveals hallmarks of circular RNA biogenesis in animals, Cell Rep., 2015, 10, 170-177.

66 K. Husken, T. Wiesenfahrt, C. Abraham, R. Windoffer, O. Bossinger and R. E. Leube, Maintenance of the intestinal tube in Caenorhabditis elegans: the role of the intermediate filament protein IFC-2, Differentiation, 2008, 76, 881-896. 
67 S. J. Conn, K. A. Pillman, J. Toubia, V. M. Conn, M. Salmanidis, C. A. Phillips, et al., The RNA binding protein quaking regulates formation of circRNAs, Cell, 2015, 160, 1125-1134.

68 A. Rybak-Wolf, C. Stottmeister, P. Glazar, M. Jens, N. Pino, S. Giusti, et al., Circular RNAs in the mammalian brain are highly abundant, conserved, and dynamically expressed, Mol. Cell, 2015, 58, 870-885.

69 Q. Zheng, C. Bao, W. Guo, S. Li, J. Chen, B. Chen, et al., Circular RNA profiling reveals an abundant circHIPK3 that regulates cell growth by sponging multiple miRNAs, Nat. Commun., 2016, 7, 11215. 